\title{
INTEGRAL Observations of GW170104
}

\section{Savchenko, V.; Ferrigno, C.; Bozzo, E.; Bazzano, A.; Brandt, Søren; Chenevez, Jérôme; Courvoisier, T. J.-L.; Diehl, R.; Hanlon, L.; von Kienlin, A.}

Total number of authors:

19

Published in:

The Astrophysical Journal Letters

Link to article, DOI:

10.3847/2041-8213/aa87ae

Publication date:

2017

Document Version

Publisher's PDF, also known as Version of record

Link back to DTU Orbit

Citation (APA):

Savchenko, V., Ferrigno, C., Bozzo, E., Bazzano, A., Brandt, S., Chenevez, J., Courvoisier, T. J-L., Diehl, R., Hanlon, L., von Kienlin, A., Kuulkers, E., Laurent, P., Lebrun, F., Lutovinov, A. A., Martin-Carillo, A., Mereghetti, S., Roques, J-P., Sunyaev, R., \& Ubertini, P. (2017). INTEGRAL Observations of GW170104. The Astrophysical Journal Letters, 846(2), [L23]. https://doi.org/10.3847/2041-8213/aa87ae

\section{General rights}

Copyright and moral rights for the publications made accessible in the public portal are retained by the authors and/or other copyright owners and it is a condition of accessing publications that users recognise and abide by the legal requirements associated with these rights.

- Users may download and print one copy of any publication from the public portal for the purpose of private study or research.

- You may not further distribute the material or use it for any profit-making activity or commercial gain

- You may freely distribute the URL identifying the publication in the public portal 


\title{
INTEGRAL Observations of GW170104
}

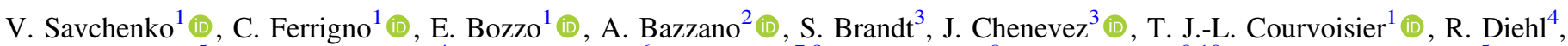 \\ L. Hanlon $^{5}$ (D), A. von Kienlin ${ }^{4}$ (D), E. Kuulkers ${ }^{6}$, P. Laurent ${ }^{7,8}$ (D), F. Lebrun ${ }^{8}$, A. Lutovinov ${ }^{9,10}$ (D), A. Martin-Carillo ${ }^{5}$, \\ S. Mereghetti ${ }^{11}$ (D), J. P. Roques ${ }^{12}$, R. Sunyaev ${ }^{9,13}$, and P. Ubertini ${ }^{3}$ \\ ${ }^{1}$ ISDC, Department of Astronomy, University of Geneva, chemin d'Écogia, 16 CH-1290 Versoix, Switzerland \\ 2 INAF-Institute for Space Astrophysics and Planetology, Via Fosso del Cavaliere 100, I-00133-Rome, Italy \\ ${ }^{3}$ DTU Space-National Space Institute Elektrovej, Building 327, DK-2800 Kongens Lyngby, Denmark \\ ${ }^{4}$ Max-Planck-Institut für Extraterrestrische Physik, Garching, Germany \\ ${ }^{5}$ Space Science Group, School of Physics, University College Dublin, Belfield, Dublin 4, Ireland \\ ${ }^{6}$ European Space Research and Technology Centre (ESA/ESTEC), Keplerlaan 1, 2201 AZ Noordwijk, The Netherlands \\ ${ }^{7}$ APC, AstroParticule et Cosmologie, Université Paris Diderot, CNRS/IN2P3, CEA/Irfu, Observatoire de Paris Sorbonne Paris Cité, \\ 10 rue Alice Domont et Léonie Duquet, F-75205 Paris Cedex 13, France \\ ${ }^{8}$ DSM/Irfu/Service d'Astrophysique, Bat. 709 Orme des Merisiers CEA Saclay, F-91191 Gif-sur-Yvette Cedex, France \\ ${ }^{9}$ Space Research Institute of Russian Academy of Sciences, Profsoyuznaya 84/32, 117997 Moscow, Russia \\ ${ }^{10}$ Moscow Institute of Physics and Technology, Institutskiy per. 9, Dolgoprudny, Moscow Region, 141700, Russia \\ ${ }^{11}$ INAF, IASF-Milano, via E.Bassini 15, I-20133 Milano, Italy \\ ${ }^{12}$ Université Toulouse, UPS-OMP, CNRS, IRAP, 9 Av. Roche, BP 44346, F-31028 Toulouse, France \\ ${ }^{13}$ Max Planck Institute for Astrophysics, Karl-Schwarzschild-Str. 1, D-85741 Garching b. Munchen, Germany \\ Received 2017 July 12; revised 2017 August 16; accepted 2017 August 18; published 2017 September 6
}

\begin{abstract}
We used data from the International Gamma-Ray Astrophysics Laboratory (INTEGRAL) to set upper limits on the $\gamma$-ray and hard X-ray prompt emission associated with the gravitational-wave event GW170104, discovered by the Laser Interferometer Gravitational-wave Observatory (LIGO)/Virgo collaboration. The unique omnidirectional viewing capability of the instruments on board INTEGRAL allowed us to examine the full $90 \%$ confidence level localization region of the LIGO trigger. Depending on the particular spectral model assumed and the specific position within this region, the upper limits inferred from the INTEGRAL observations range from $F_{\gamma}=1.9 \times 10^{-7} \mathrm{erg} \mathrm{cm}^{-2}$ to $F_{\gamma}=10^{-6} \mathrm{erg} \mathrm{cm}^{-2}$ (75 keV-2 MeV energy range). This translates into a ratio between the prompt energy released in $\gamma$-rays along the direction to the observer and the gravitational-wave energy of $E_{\gamma} / E_{\mathrm{GW}}<2.6 \times 10^{-5}$. Using the INTEGRAL results, we cannot confirm the $\gamma$-ray proposed counterpart to GW170104 by the Astro-Rivelatore Gamma a Immagini Leggero (AGILE) team with the mini-Calorimeter (MCAL) instrument. The reported flux of the AGILE/MCAL event, E2, is not compatible with the INTEGRAL upper limits within most of the $90 \%$ LIGO localization region. There is only a relatively limited portion of the sky where the sensitivity of the INTEGRAL instruments was not optimal and the lowest-allowed fluence estimated for E2 would still be compatible with the INTEGRAL results. This region was also observed independently by Fermi/Gamma-ray Burst Monitor and AstroSAT, from which, as far as we are aware, there are no reports of any significant detection of a prompt high-energy event.
\end{abstract}

Key words: gamma-ray burst: general - gravitational waves

\section{Introduction}

The Laser Interferometer Gravitational-wave Observatory (LIGO)/Virgo collaboration reported a third significant gravitational-wave (GW) event, GW170104, discovered on 2017 January 4 10:11:58.6 UTC. The false-alarm probability associated with the detection was less than one event over 70,000 years (Abbott et al. 2017). The LIGO 90\% confidence localization region of GW170104 consisted of two elongated arcs, each spanning over $120^{\circ}$. The event was associated with the merging of two black holes with masses of $31_{-6}^{+8.4} M_{\odot}$ and $19_{-5.9}^{+5.3} M_{\odot}$ at a distance of $880_{-390}^{+450} \mathrm{Mpc}$. GW170104 is thus the most remote confirmed GW event discovered so far.

Following the announcement by the LIGO team, extensive follow-up observations were carried out by a large number of facilities to search for an electromagnetic counterpart. Results obtained from ongoing serendipitous observations were promptly reported as well. The two telescopes on board the Fermi satellite could not detect any significant excess over the background that was spatially and temporally compatible with the GW event (Burns et al. 2017; Fermi GBM \& Fermi LAT Collaborations 2017). Fermi-Gamma-ray Burst Monitor (GBM) provided sky coverage of $69.5 \%$ at the time of GW170104, enclosing $82.4 \%$ of the LIGO localization region. The upper limit derived from the Fermi-GBM observations corresponds to a $1 \mathrm{~s}$ fluence spanning from $5.2 \times 10^{-7} \mathrm{erg} \mathrm{cm}^{-2}$ to $9.4 \times 10^{-7} \mathrm{erg} \mathrm{cm}^{-2}$ (in the $8-1000 \mathrm{keV}$ energy range and assuming a typical Band spectrum of a short $\gamma$-ray burst, GRB). A tighter upper limit on the fluence of the event was reported by AstroSAT in a more restricted region of the sky (Bhalerao et al. 2017). A nondetection at the $95 \%$ confidence level (c.l.) was also reported by Konus-Wind (Svinkin et al. 2017b).

One of the instruments on board the Astro-Rivelatore Gamma a Immagini Leggero (AGILE) satellite revealed an excess over the instrument background (AGILE-GW170104) that was roughly coincident in time with the GW event. The estimated signal to noise ratio $(\mathrm{S} / \mathrm{N})$ of the detection is 4.4 and the corresponding post-trial coincidence probability is between $2.4 \sigma$ and $2.7 \sigma$ (Verrecchia et al. 2017).

In this Letter, we make use of the available data collected by the instruments on board the International Gamma-Ray Astrophysics Laboratory (INTEGRAL; Winkler et al. 2003) to search for possible hard X-ray and $\gamma$-ray counterparts to GW170104. We summarize the most relevant capabilities of the INTEGRAL instruments for these kinds of searches in 
Section 2 and describe all the obtained results in Section 3. We discuss the nondetection of a counterpart to the GW event in the INTEGRAL data with respect to the findings reported by the AGILE team in Section 3.1. Our conclusions are reported in Section 4.

\section{The INTEGRAL Instruments and the Follow-up of GW Events}

As extensively described by Savchenko et al. (2017), INTEGRAL provides unique instantaneous coverage of the entire high-energy sky by taking advantage of the synergy between its four all-sky detectors: IBIS/ISGRI, IBIS/PICsIT, IBIS/Veto, and SPI-ACS. These provide complementary capabilities for the detection of transient events characterized by different durations, locations on the sky, and spectral energy distributions. In the case of the first GW event, GW150914, the most stringent upper limit on the nondetection of an electromagnetic counterpart in the $75 \mathrm{keV}$ to $2 \mathrm{MeV}$ energy range with INTEGRAL was obtained with the SPI-ACS (Savchenko et al. 2016), while the peculiar localization of LVT151012 (Abbott et al. 2016) and its orientation with respect to the INTEGRAL satellite required the combination of the results from all detectors (together with a careful analysis of each instrument's response and background) to achieve an optimized upper limit. As we discuss in Section 3, it is again the SPI-ACS that provides the most stringent upper limit on the high-energy emission from the nondetected electromagnetic counterpart to GW170104.

The SPI-ACS (von Kienlin et al. 2003) is made of 91 BGO (Bismuth Germanate, $\mathrm{Bi}_{4} \mathrm{Ge}_{3} \mathrm{O}_{12}$ ) scintillator crystals and it is the anti-coincidence shield of the SPI instrument (Vedrenne et al. 2003). Besides its main function of shielding the SPI germanium detectors, the ACS is also used as a nearly omnidirectional detector of transient events, providing a large effective area at energies above $\sim 75 \mathrm{keV}$. The ACS data consist of event rates integrated over all the scintillator crystals with a time resolution of $50 \mathrm{~ms}$. No spectral and/or directional information of the recorded events is available. The typical number of counts per $50 \mathrm{~ms}$ time bin ranges typically from about 3000 to 6000 . SPI-ACS features a high duty cycle of $\sim 85 \%{ }^{14}$ and comprises events from the nearly complete highenergy sky.

SPI is partially surrounded by the satellite structure and by the other INTEGRAL instruments, which shield the incoming photons and thus also affect the response of the ACS in different directions. For this reason, the computation of the ACS response requires detailed simulations that take into account the entire satellite structure. We developed a GEANT3 Monte Carlo model based on the INTEGRAL mass model (Sturner et al. 2003) and simulated the propagation of monochromatic parallel beams of photons in the $50 \mathrm{keV}-$ $100 \mathrm{MeV}$ energy range. For each energy, we simulated 3072 sky positions (16-sided HEALPix ${ }^{15}$ grid). This allows us to generate an instrumental response function for any position in the sky, which can then be used to compute the expected number of counts for a given source spectral energy distribution. As shown in our previous paper (Savchenko

\footnotetext{
${ }^{14}$ The reduction of $15 \%$ is due to the fact that the INTEGRAL instruments are switched off near the perigee of every satellite revolution. The INTEGRAL orbit was as long as three sidereal days until 2015 January, but was later shortened to 2.7 to allow for a safe satellite disposal in 2029 .

15 http://healpix.sourceforge.net
}

et al. 2017), this response produces results for the bursts detected simultaneously by the SPI-ACS and other detectors (Fermi/GBM and Konus-Wind) that are consistent to an accuracy better than $20 \%$.

\section{INTEGRAL Observations of GW170104}

At the time of the GW170104 (2017 January 4 10:11:58.6 UTC, hereafter $T_{0}$ ), INTEGRAL was fully operational and executing the pointing ID. 176700040010 in the direction of the Cas A/Tycho supernova remnants, far from the likely localization region of the LIGO trigger. All instruments were performing nominally, yielding a virtually constant and stable background count rate from at least $T_{0}-2500$ to $T_{0}+2500$ ks. The SPI-ACS background count rate was about $1.14 \times 10^{5}$ counts $\mathrm{s}^{-1}$, which is higher than that observed during LVT151012 or GW150914 and close to the maximum value ever observed in SPI-ACS data during the INTEGRAL mission lifetime (excluding the time intervals affected by solar flares). There are two reasons for the high background recorded at the time of GW170104: the 11 year solar activity cycle, which is close to its minimum, and the day-scale variations of the instrumental background, which have been commonly observed since the early stages of the instrument operations. The enhanced background rate decreases the sensitivity of INTEGRAL instruments by as much as $30 \%$, when compared to the most favorable conditions and much less, when compared to our reports on LVT151012 and GW150914. However, it should be noted that the effects of background fluctuations on the sensitivity are typically smaller than those due to sky location. At the time of GW170104, the Earth was relatively distant from INTEGRAL, casting a small shadow of $49.0 \mathrm{deg}^{2}$ on the instrument field of view (FoV; equivalent to $0.12 \%$ of the sky) and occulting only about $0.032 \%$ of LIGO event localization probability. In the remaining part of this region, the SPI-ACS sensitivity was close to optimal. Thus, this instrument allowed us to carry out the most accurate search for any electromagnetic counterpart to GW170104. For a fraction of the $90 \%$ LIGO localization region, the IBIS sensitivity, including both ISGRI and PICsIT, (Ubertini et al. 2003) approached that of the SPI-ACS, but we checked that adding these data did not significantly improve our results. Therefore, we do not extensively comment on the IBIS data but report for completeness in Figure 1 a comparison between the contributions provided by the SPI-ACS, IBIS/Veto, and ISGRI in searching for an electromagnetic counterpart of GW170104. In this figure, we estimated for each value of the upper limit the integrated fraction of the entire LIGO localization region of the GW event that is probed by the data of the different INTEGRAL instruments. The SPI-ACS is clearly able to provide the deepest limits in the entire portion of the sky where the LIGO localization probability is significantly larger than zero.

The INTEGRAL Burst Alert System (IBAS; Mereghetti et al. 2003) routinely inspects the INTEGRAL SPI-ACS and IBIS/ ISGRI light curves in real time, searching for significant deviations from the background and producing automatic triggers. The closest IBAS trigger to GW170104 occurred on 2017 January $422: 12: 40\left(T_{0}+43241 \mathrm{~s}\right)$ and was classified as a cosmic-ray event, thus unlikely to be related to the LIGO trigger.

The closest event identified as a possible GRB in INTEGRAL data occurred at 2017 January 5 06:14:06 with an S/N of 9.3 and a duration of $5 \mathrm{~s}$. The astrophysical nature of this event was 


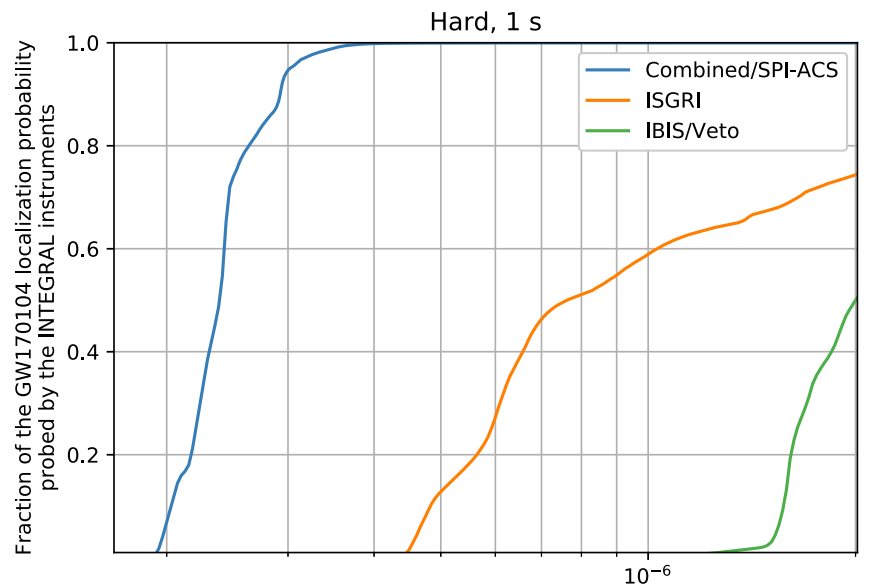

upper limit, erg $\mathrm{cm}^{-2}, 75-2000 \mathrm{keV}$

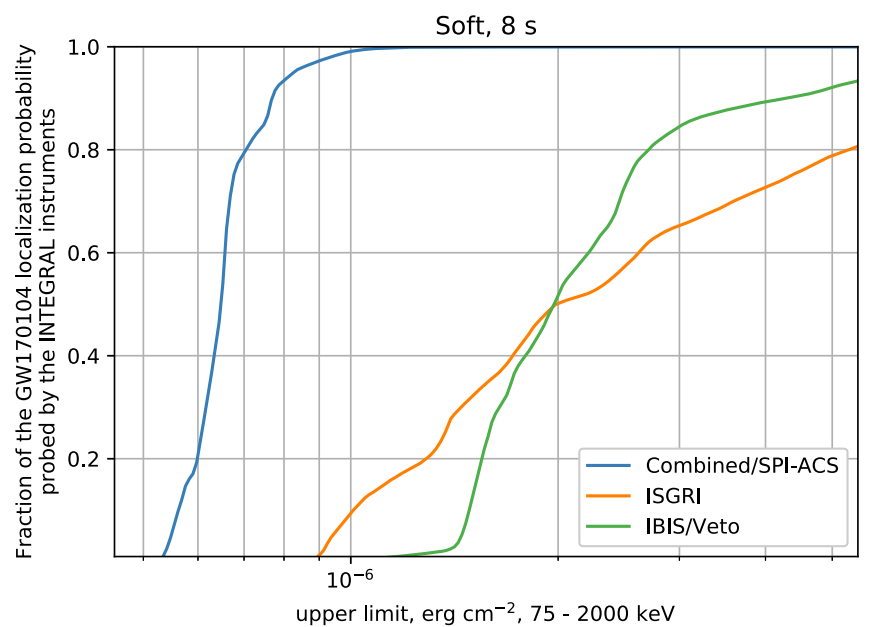

Figure 1. Plot of the fraction of the LIGO localization probability of GW170104 probed by the data of the different INTEGRAL instruments as a function of the upper limit ( $3 \sigma$ c.l.) on the nondetected electromagnetic counterpart to the GW event. The left panel is for the case of the short-hard burst, while the right panel shows the case of a long-soft burst (see the text for details). The "Combined/SPI-ACS" text in the label indicates that the results do not quantitatively change if only the SPI-ACS data are used to draw the blue solid line or if the independent contributions from the other instruments are also merged.

confirmed by simultaneous observations of Konus-Wind (Svinkin et al. 2017a), AstroSAT (Sharma et al. 2017), POLAR (Marcinkowski \& Xiao 2017), and a combined IPN analysis (Svinkin et al. 2017a). This was classified as a regular long GRB (GRB170105) with an optical afterglow that could also be independently found in the ATLAS follow-up observations of GW170104 (ATLAS17aeu; Bhalerao et al. 2017; Melandri et al. 2017; Stalder et al. 2017; Tonry et al. 2017). INTEGRAL observations contributed to the triangulation, which allowed for establishing the association between GRB170105 and ATLAS17aeu (Svinkin et al. 2017a). In general, INTEGRAL data are particularly useful to retrospectively search for GRB events, owing to its competitive and consistent omnidirectional sensitivity, stable background, and high duty cycle (see, e.g., a recent case studied by Whitesides et al. 2017). GRB170105 was later found to be likely unrelated to GW170104 (Bhalerao et al. 2017; Stalder et al. 2017).

We also inspected the SPI-ACS and IBIS light curves, focusing on a time interval of $\pm 500 \mathrm{~s}$ around $T_{0}$ and probing five different timescales in the range $0.05-100 \mathrm{~s}$. The latter were selected to be representative of the dynamical timescale of the accretion occurring in a coalescing compact binary (e.g., Lee \& Ramirez-Ruiz 2007). We did not find any obvious detection of a significant signal temporally coincident with the GW event. A zoom of the SPI-ACS light curve around the time of the LIGO trigger is shown in Figure 2.

Following the approach in Savchenko et al. $(2016,2017)$ and the nondetection of any significant electromagnetic counterpart to GW170104 in the INTEGRAL data, we derived the corresponding upper limits assuming the cases of (i) a shorthard burst, i.e., a $1 \mathrm{~s}$ long event characterized by a cutoff power-law spectral energy distribution with parameters $\alpha=-0.5, E_{\text {peak }}=600 \mathrm{keV}$; (ii) a long-soft burst, i.e., an $8 \mathrm{~s}$ long event whose spectral energy distribution is described by the Band model (Band et al. 1993) with parameters $\alpha=-1$, $\beta=-2.5$, and $E_{\text {peak }}=300 \mathrm{keV}$. While the reference short GRB duration of $1 \mathrm{~s}$ is close to the peak of the short GRB duration distribution, the $8 \mathrm{~s}$ timescale for the long-soft GRB is motivated by the sampling rate of IBIS/Veto, in analogy with the approach presented previously by Savchenko et al. (2017).

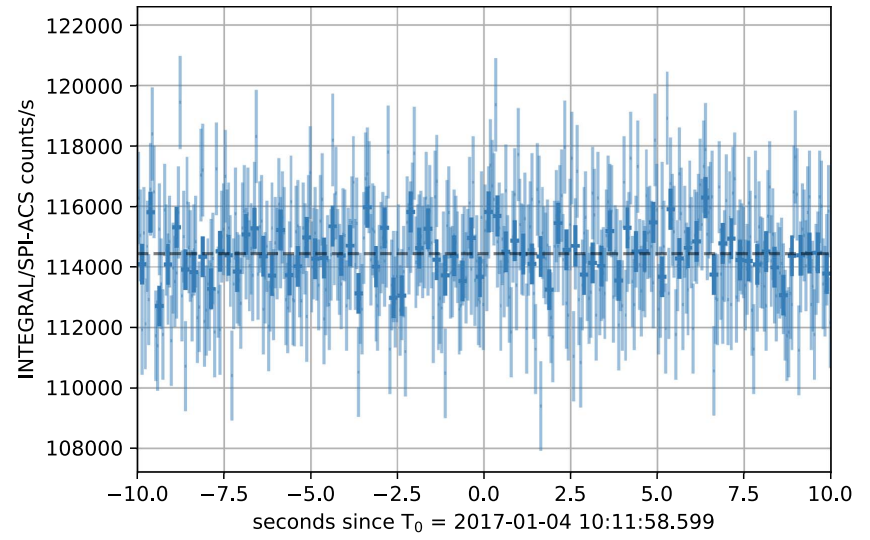

Figure 2. Zoom of the INTEGRAL/SPI-ACS light curve in the $\pm 10 \mathrm{~s}$ time interval around the LIGO detection of GW170104. Light blue symbols represent the measurements at the natural instrument time resolution of $50 \mathrm{~ms}$, while dark blue points correspond to the data rebinned at a resolution of 250 ms. The dashed black curve represents the average instrument background obtained from a much longer span of data.

To calculate the $3 \sigma$ upper limits, we fold the spectral model through the instrument response for each sky location and adjust the model normalization until the predicted number of counts is equal to three times the standard deviation of the background counts in the considered time interval. The upper limit derived in this way corresponds also to the $3 \sigma$ detection threshold, which is the generally recommended approach to compute upper limits corresponding to the nondetection of astrophysical events (Kashyap et al. 2010). Our method complies to the commonly accepted upper limit definitions, used for example by the Fermi/ GBM team (Fermi GBM \& Fermi LAT Collaborations 2017). The results obtained in these two cases are shown in Figures 3 and 4. The estimated upper limits $(75 \mathrm{keV}-2 \mathrm{MeV})$ within the LIGO $90 \%$ localization region range from $F_{\gamma}=1.9 \times 10^{-7} \mathrm{erg} \mathrm{cm}^{-2}$ to $3.5 \times 10^{-7} \mathrm{erg} \mathrm{cm}^{-2}$ for a $1 \mathrm{~s}$ short-hard GRB and from $F_{\gamma}=5.2 \times 10^{-7} \mathrm{erg} \mathrm{cm}^{-2}$ to $10^{-6} \mathrm{erg} \mathrm{cm}^{-2}$ for an $8 \mathrm{~s}$ event characterized by a typical long GRB spectrum.

Assuming the reference distance to the event of $D=880 \mathrm{Mpc}$ (Abbott et al. 2017), we can derive an upper limit on the isotropic equivalent total energy released in the 75-2000 keV energy band 
INTEGRAL 3-sigma upper limit during GW170104 (short-hard burst)

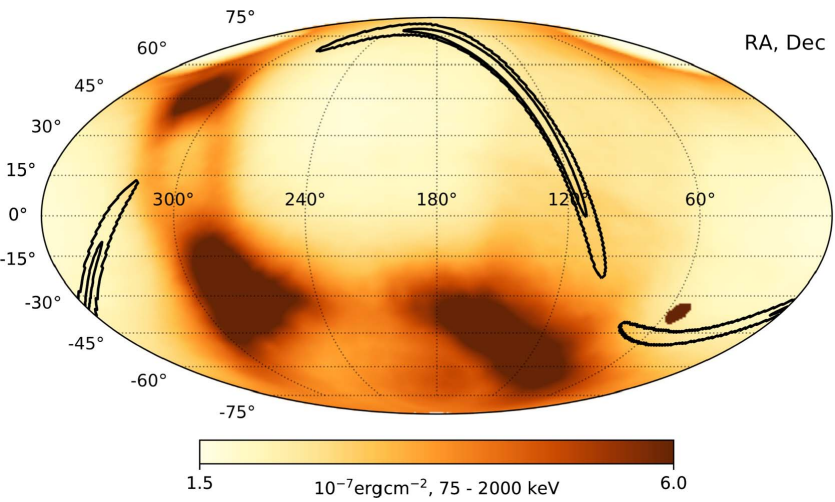

Figure 3. Estimated $3 \sigma$ upper limits on the $75-2000 \mathrm{keV}$ flux of the nondetected electromagnetic counterpart to GW170104 as derived from the SPI-ACS data assuming the case of a short-hard burst. The black contours show the most accurate localization of the GW event at $50 \%$ and $90 \%$ c.l., as provided by the LALInference (Abbott et al. 2017).

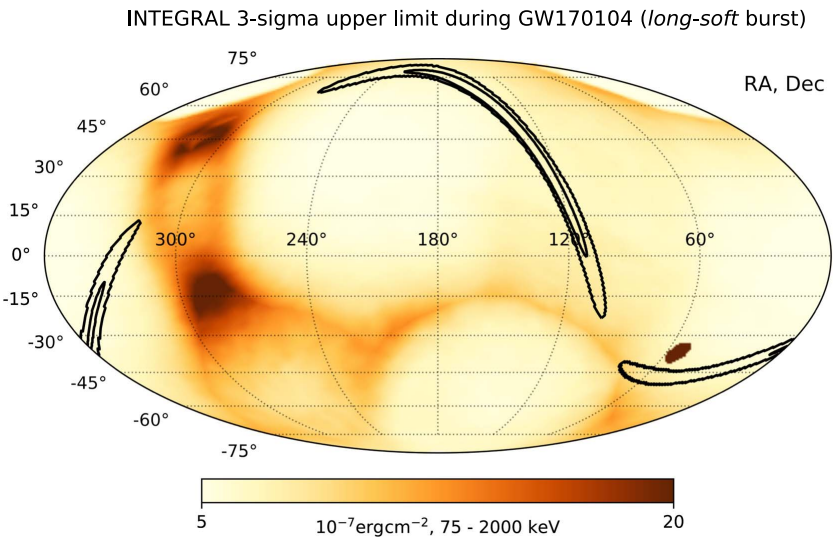

Figure 4. Same as Figure 3, but for the case of a long-soft burst.

in $1 \mathrm{~s}$ as $E_{\gamma}<3.2 \times 10^{49} \operatorname{erg}\left(\frac{F_{\gamma}}{3.5 \times 10^{-7} \mathrm{erg} \mathrm{cm}^{-2}}\right)\left(\frac{D}{880 \mathrm{Mpc}}\right)^{2}$. The energy emitted in gravitational waves can be estimated as $E_{\mathrm{GW}}=3.6_{-1.3}^{+1.1} \times 10^{54} \mathrm{erg}$. The SPI-ACS upper limits we reported above can constrain the fraction of energy emitted in hard X-rays and $\gamma$-rays toward the observer during the GW event to be $f_{\gamma}<9 \times 10^{-6}$ in the case of the short-hard burst, and $f_{\gamma}<2.6 \times 10^{-5}$ in the case of the long-soft one (in the 75-2000 keV energy range).

While the limit on the fraction of the gamma-ray energy emitted in the energy range covered by SPI-ACS has the advantage of depending the least on the assumed source spectrum, it is of a general interest to estimate a limit on the bolometric luminosity. In the $1-10,000 \mathrm{keV}$ energy range that is conventionally used (e.g., Rowlinson et al. 2014; Pescalli et al. 2016), we can constrain the total released electromagnetic energy and its ratio to the GW energy as $E_{1-10^{5} \mathrm{keV}}<3.5 \times 10^{49}$ $\left(f_{1-10^{5} \mathrm{keV}}<9.8 \times 10^{-6}\right)$ in the case of the short-hard burst, and $E_{1-10^{5} \mathrm{keV}}<1.3 \times 10^{50}\left(f_{1-10^{5} \mathrm{keV}}<3.7 \times 10^{-5}\right)$ in the case of the long-soft one.

\subsection{On the Possible AGILE Detection of an Electromagnetic Counterpart to GW170104}

AGILE is an X-ray and $\gamma$-ray astronomical satellite of The Italian Space Agency, launched in 2007. AGILE's scientific
INTEGRAL 90\% upper bound on AGILE-GW170104-E2

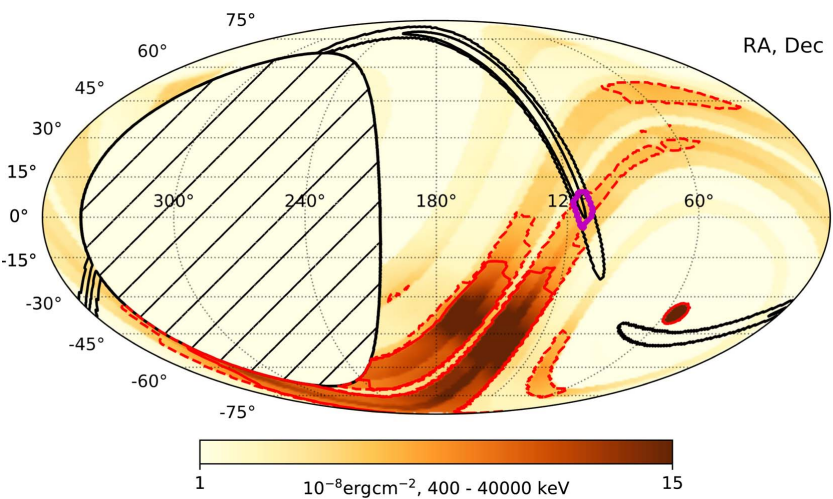

Figure 5. Plot of the estimated lowest detectable fluence at $90 \%$ c.l. by the SPI-ACS for a $32 \mathrm{~ms}$ long burst going off at the time of AGILE-E2 in different positions of the sky (a spectral energy distribution with a slope of -2 has been assumed). The large dashed circle corresponds to the location occulted for AGILE by the Earth. The small dark circle represents the region occulted by the Earth to INTEGRAL. Solid red lines enclose the regions where the lowest detectable SPI-ACS fluence is higher than the best-fit one $\left(8.9 \times 10^{-8} \mathrm{erg} \mathrm{cm}^{-2}\right)$ obtained for the tentative AGILE counterpart of GW170104 (i.e., the event E2). Dashed red lines are used for the same comparison with the lower boundary of the AGILE fluence $\left(5.9 \times 10^{-8} \mathrm{erg} \mathrm{cm}^{-2}\right)$. The thick magenta lines encircle the position of the sky within the $90 \%$ LIGO localization region of GW170104 in which the minimum fluence reported for AGILE-GW170104 is compatible with the INTEGRAL results.

payload comprises a pair-conversion telescope, capable of detecting photons in the $30 \mathrm{MeV}-100 \mathrm{GeV}$ energy range (GRID), and a hard X-ray monitor sensitive in the $18-60 \mathrm{keV}$ energy range (SuperAGILE, or SA). Additionally, AGILE is able to observe bright impulsive events from a large fraction of the unocculted sky with its mini-Calorimeter (MCAL), operating in the energy band $0.4-100 \mathrm{MeV}$ (Tavani et al. 2008).

Verrecchia et al. (2017) reported on observations carried out with the MCAL at the time of GW170104. These observations covered only a fraction of the LIGO localization, due to the occultation of the AGILE FoV caused by the Earth. Several weak bursts were identified in the AGILE/MCAL data around the time of GW170104. Among them, the $32 \mathrm{~ms}$ long burst E2 was identified as a possible $\gamma$-ray counterpart of the GW event. The reported trigger time is at $0.46 \pm 0.05 \mathrm{~s}$ before $T_{0}$.

Following the report by Verrecchia et al. (2017), we investigated the INTEGRAL data to check for any confirmation of this detection. We note that, unlike the upper limit presented in the previous section (Figures 3 and 4), we need to compute the upper bound in the flux of any possible celestial event corresponding to the measured signal in SPI-ACS at the exact time of the AGILE putative event. However, the INTEGRAL orbit is very elongated resulting in a sizable difference in a celestial signal arrival time, which depends on the unknown source sky location, reaching up to $\pm 0.32 \mathrm{~s}$. First, we computed for each position in the sky the time at which the event AGILE-GW170104 should have been observed by INTEGRAL. For each position in the sky at the proper trigger time, we show with a color map in Figure 5 the corresponding $90 \%$ c.l. values of the upper bound on the $400-40,000 \mathrm{keV}$ fluence consistent with the SPI-ACS count rate. ${ }^{16}$ The reported

\footnotetext{
16 Note that the $90 \%$ c.l. was preferred to the $3 \sigma$ approach to compare more easily the INTEGRAL and AGILE findings.
} 
values are calculated assuming a $32 \mathrm{~ms}$ long event characterized by a power-law-shaped spectral energy distribution with a slope of -2 (as done in Verrecchia et al. 2017). As SPI-ACS observes positive and negative count rate fluctuations in the background, all positions corresponding to a certain time delay between the INTEGRAL and AGILE locations define circularly shaped regions in the sky within which the upper bound on the event flux is constant. This is the reason why the map of the upper bound values in Figure 5 comprises stripes of different colors. The source positions in the sky coincident with the direction toward AGILE as seen from INTEGRAL and the diametrically opposite direction correspond to the maximum absolute time delays. Since the altitude of AGILE's orbit is much smaller than that of INTEGRAL's orbit, the direction from INTEGRAL toward AGILE is very close to the direction from INTEGRAL to Earth, and the circularly shaped regions are all approximately centered on the position of the Earth (a small dark circle in Figure 5). The median value of the fluence in sky locations compatible to the time delay between the spacecraft is $1.7 \times 10^{-8} \mathrm{erg} \mathrm{cm}^{-2}$ and it does not exceed $7.1 \times$ $10^{-8} \mathrm{erg} \mathrm{cm}^{-2}$ in any sky position enclosed within the LIGO 90\% localization region of GW170104. In Figure 5, we highlighted with red contours the portions of the sky where the minimum detectable fluence by INTEGRAL is consistent with the best-fit (solid) and the lowest-allowed (dashed) fluence of AGILE-GW170104 inferred from the AGILE data.

We found that there are no sky positions within the $90 \%$ LIGO localization region for which the best-fit fluence of the AGILE event is compatible with the INTEGRAL results. There are, however, positions within the 90\% LIGO localization region for which the lowest-allowed value of the fluence of AGILE-GW170104 would still be compatible with the INTEGRAL results (thick magenta contour in Figure 5). The ensemble of these positions covers about $4.2 \%$ of the LIGO localization region and extends for a total of $77.5 \mathrm{deg}^{2}$. Note that a few small regions enclosed within red dashed lines are sparsely present in the color map of Figure 5. These are positions in the sky for which the AGILE trigger time of AGILE-GW170104 corresponds to positive count rate fluctuations in the SPI-ACS light curve. We inspected each of these fluctuations, but none of them exceeded an $\mathrm{S} / \mathrm{N}$ of 1.5.

Taking together all these results, we cannot exclude that the event AGILE-GW170104 is associated with the GW trigger if it originated from a restricted number of positions in the sky within the 90\% LIGO localization region. However, this detection is compatible with the INTEGRAL results only if a fluence that is a factor of 1.2 lower than the best-fit value obtained from the AGILE data is considered.

We noticed that the limited positions in the sky within the 90\% LIGO localization region for which the AGILE/MCAL detection is compatible with the INTEGRAL results were also accessible to the Fermi/GBM (Burns et al. 2017; Fermi GBM \& Fermi LAT Collaborations 2017) and, in an even more limited way, by the AstroSAT/CZTI (Bhalerao et al. 2017). Further analysis of the observations performed by these two facilities could help to confirm the AGILE detection.

The conclusions above depend significantly on the assumed spectral energy distribution of the event. A detailed description of the spectral parameters of AGILE-GW170104 is not provided by Verrecchia et al. (2017), and thus we followed their assumption of a power-law-shaped energy distribution with a slope of -2 . At the same time, the authors also indicated that AGILE-GW170104 features similar timing and spectral properties to the precursor of GRB090510. This weak precursor was detected by both AGILE/MCAL and Fermi/ GBM. It was also detected by INTEGRAL/SPI-ACS with an $\mathrm{S} / \mathrm{N}$ of 6.1 , even though the location in the sky was not covered with the optimal sensitivity of the SPI-ACS. By analyzing the response of this instrument in the direction of GRB090510 and using the results obtained from the observation of the precursor of the GRB, we were able to derive a nearly model-independent conclusion that a similar event occurring anywhere within the LIGO 90\% localization region of GW170104, excluding the area invisible to AGILE, should have been detected by the SPI-ACS with a median $\mathrm{S} / \mathrm{N}$ of 13.0, and certainly no lower than 4.6.

Finally, we stress that it is entirely possible that the AGILE/ MCAL event was a real weak short GRB going off in a region of the sky covered with a low SPI-ACS sensitivity and completely unrelated to GW170104 (i.e., outside the $90 \%$ LIGO localization region). Combining the area of the sky with unfavorable orientations for the SPI-ACS observations and not occulted by the Earth for AGILE, we inferred a remaining allowed region spanning about $3533 \mathrm{deg}^{2}$.

\section{Conclusions}

All GW events reported so far by LIGO were found to be most likely associated with binary back hole mergers. The extensive multi-wavelength follow-up campaigns carried out after each of these discoveries led to the detection of at least two possible electromagnetic counterparts to the GW events (Connaughton et al. 2016; Greiner et al. 2016; Verrecchia et al. 2017). Although none of these associations was firmly confirmed, they led to discussion of exotic scenarios in explaining electromagnetic emission in these mergers (e.g., Loeb 2016; Lyutikov 2016; Perna et al. 2016; Woosley 2016). The INTEGRAL efforts to follow up as much as possible all relevant LIGO triggers will eventually help to reveal which, if any, of these scenarios is applicable. So far, the INTEGRAL results have provided the most stringent upper limits on any associated prompt hard X-ray and $\gamma$-ray emission in the $75 \mathrm{keV}$ to $2 \mathrm{MeV}$ energy range for each of the announced $\mathrm{GW}$ events when INTEGRAL observations were available, challenging the possible association of GW150914 and GW170104 with the tentatively reported electromagnetic counterparts.

Based on observations with INTEGRAL, an ESA project with instruments and science data center funded by ESA member states (especially the PI countries: Denmark, France, Germany, Italy, Switzerland, Spain), and with the participation of Russia and the USA. The SPI-ACS detector system has been provided by MPE Garching/Germany. We acknowledge the German INTEGRAL support through DLR grant 50 OG 1101. The Italian INTEGRAL/IBIS team acknowledges the support of ASI/INAF agreement No. 2013-025-R.0. A.L. and R.S. acknowledge support from the Russian Science Foundation (grant 14-22-00271). Some of the results in this Letter have been derived using the HEALPix (Górski et al. 2005) package. We are grateful the François Arago Centre at APC for providing computing resources, and VirtualData from LABEX P2IO for enabling access to the StratusLab academic cloud. Finally, we thank the anonymous referee for the insightful comments. 


\section{ORCID iDs}

V. Savchenko (1) https://orcid.org/0000-0001-6353-0808

C. Ferrigno (1) https://orcid.org/0000-0003-1429-1059

E. Bozzo 누 https://orcid.org/0000-0002-7504-7423

A. Bazzano (1) https://orcid.org/0000-0002-2017-4396

J. Chenevez (1) https://orcid.org/0000-0002-4397-8370

T. J.-L. Courvoisier (10 https://orcid.org/0000-0003-2396-6249

L. Hanlon (ㄴ) https://orcid.org/0000-0003-2931-3732

A. von Kienlin (1) https://orcid.org/0000-0002-0221-5916

P. Laurent $\odot$ https://orcid.org/0000-0001-9094-0335

A. Lutovinov (1) https://orcid.org/0000-0002-6255-9972

S. Mereghetti (1) https://orcid.org/0000-0003-3259-7801

\section{References}

Abbott, B. P., Abbott, R., Abbott, T. D., et al. 2016, PhRvX, 6, 041015 Abbott, B. P., Abbott, R., Abbott, T. D., et al. 2017, PhRvL, 118, 221101 Band, D., Matteson, J., Ford, L., et al. 1993, ApJ, 413, 281

Bhalerao, V., Kasliwal, M. M., Bhattacharya, D., et al. 2017, ApJ, 845, 152

Burns, E., Blackburn, L., Briggs, M. S., et al. 2017, GCN, 20365, https://gcn. gsfc.nasa.gov/gen3/20365.gcn3

Connaughton, V., Burns, E., Goldstein, A., et al. 2016, ApJL, 826, L6

Fermi GBMFermi LAT Collaborations 2017, arXiv:1706.00199

Górski, K., Hivon, E., Banday, A., et al. 2005, ApJ, 622, 759

Greiner, J., Burgess, J. M., Savchenko, V., \& Yu, H.-F. 2016, ApJL, 827, L38

Kashyap, V. L., Van Dyk, D. A., Connors, A., et al. 2010, ApJ, 719, 900

Lee, W., \& Ramirez-Ruiz, E. 2007, NJPh, 9, 17
Loeb, A. 2016, ApJL, 819, L21

Lyutikov, M. 2016, arXiv: 1602.07352

Marcinkowski, R., \& Xiao, H. 2017, GCN, 20387

Melandri, A., Piranomonte, S., Branchesi, M., et al. 2017, GCN, 20735

Mereghetti, S., Götz, D., Borkowski, J., Walter, R., \& Pedersen, H. 2003, A\&A, 411, L291

Perna, R., Lazzati, D., \& Giacomazzo, B. 2016, ApJL, 821, L18

Pescalli, A., Ghirlanda, G., Salvaterra, R., et al. 2016, A\&A, 587, A40

Rowlinson, A., Gompertz, B. P., Dainotti, M., et al. 2014, MNRAS, 443, 1779

Savchenko, V., Bazzano, A., Bozzo, E., et al. 2017, A\&A, 603, A46

Savchenko, V., Ferrigno, C., Mereghetti, S., et al. 2016, ApJL, 820, L36

Sharma, V., Bhalerao, V., Bhattacharya, D., \& Rao, A. R. 2017, GCN, 20389

Stalder, B., Tonry, J., Smartt, S. J., et al. 2017, ApJ, submitted (arXiv:1706. 00175)

Sturner, S., Shrader, C., Weidenspointner, G., et al. 2003, A\&A, 411, L81

Svinkin, D., Golenetskii, S., Aptekar, R., et al. 2017a, GCN, 20406, https:// gcn.gsfc.nasa.gov/gcn3/20406.gcn3

Svinkin, D., Golenetskii, S., Aptekar, R., et al. 2017b, GCN, 21158, https:// gcn.gsfc.nasa.gov/gcn3/21158.gcn3

Tavani, M., Collaboration, F. T. A., Argan, A., et al. 2008, A\&A, 502, 995

Tonry, J., Denneau, L., Heinze, A., et al. 2017, GCN, 20377, https://gcn.gsfc. nasa.gov/gen3/20377.gcn3

Ubertini, P., Lebrun, F., Di Cocco, G., et al. 2003, A\&A, 411, L131

Vedrenne, G., Roques, J.-P., Schönfelder, V., et al. 2003, A\&A, 411, L63

Verrecchia, F., Tavani, M., Ursi, A., et al. 2017, ApJL, submitted (arXiv:1706. 00029)

von Kienlin, A., Beckmann, V., Rau, A., et al. 2003, A\&A, 411, L299

Whitesides, L., Lunnan, R., Kasliwal, M. M., et al. 2017, ApJ, submitted (arXiv:1706.05018)

Winkler, C., Courvoisier, T. J.-L., di Cocco, G., et al. 2003, A\&A, 411, L1

Woosley, S. E. 2016, ApJL, 824, 10 